\title{
Estudio de la marcha en la infancia
}

\author{
RODRIGUEZ TORRES, R.; DANKLOFF MORA, C.; GOMEZ PELLICO, L.
}

\section{RESUMEN}

El análisis de la marcha se ha visto reforzado por la aparición de sistemas de invetigación de nueva tecnología como son las plataformas de fuerzas monitorizadas que permiten estudiar, con alta precisión, el desarrollo cinético y en parte cinemático de la marcha. Con este sistema hemos estudiado el paso de 62 niños de 5 y 6 años de edad, para determinar los valores de normalidad de los parámetros temporoespaciales y las magnitudes de las fuerzas de reacción pie-suelo en los tres ejes de plano espaciales.

Hemos encontrado diferencias estadísticamente significativas entre estas edades para la longitud de paso, tiempo de doble apoyo y la mayoria de las fuerzas de reacción del suelo, indicando una evolución de la marcha en este periodo.

\section{SUMMARY}

Gait analysis has strengthened by the appearance of research systems with new and improved technology, like monitorized force platforms, which allow to study with high accuracy the kinetic and partly kinematic gait development.

With this system, we have studied the gait of 62 children aged between 5 and 6 years, in order to determine the normal values of temporal and spatial parameters, and the magnitudes of foot-ground reaction forces at the three space axes.

We found significative statistical differences between this ages for step length, double support time, and the most ground reaction forces, showing an evolution of gait in this period.

\section{INTRODUCCION}

En los últimos años el avance tecnológico está permitiendo analizar en profundidad las características que presenta la marcha humana. Uno de los campos que requiere un mayor esfuerzo es el estudio de dicha actividad en los niños, dado que el proceso de la marcha es un fenómeno complejo que precisa una fase de aprendizaje en las que establecen las pautas de su desarrollo. E1 conocimiento del mismo, permitirá avanzar con objetividad en una faceta de la antropología tan importante como es la locomoción humana.

El análisis del movimiento puede realizarse desde tres puntos de vista:
1. Cinemático: Para la descripción de los desplazamientos que sufre un cuerpo en función del tiempo.

2. Cinético: Que analiza las causas físicas que producen el movimiento.

3. Energético y metabólico: Que determina el gasto producido y estudia los procesos bioquímicos que 10 determinan.

A través de diversos métodos podemos seguir la evolución del aprendizaje de la marcha perfeccionando nuestros conocimientos. Entre las técnicas actuales para evaluar objetivamente la locomoción humana destacan las plataformas dinamométricas al ser cuantitativas y exactas (Herzog y cols. 1989; Begg 19900 ellas analizan los patrones de contacto pie-suelo durante la marcha. Beck y cols. (1981) ya señalaron que las fuerzas de reacción del suelo y los factores temporoespaciales pueden ser útiles para valorar el desarrollo de los patrones de marcha de los niños en crecimiento.

Nuestro objetivo principal fue establecer los valores de normalidad para los patrones temporoespaciales y las fuerzas de reacción del suelo con plataformas dianamométricas en la macha de niños de 5 y 6 años de edad.

\section{MATERIAL Y MÉTODO}

Hemos estudiado la marcha de 62 niños de ambos sexos sin patología del sistema locomotor, separando dos grupos según la edad, un grupo de 5 años y otro de 6 años. Cada grupo estaba compuesto de 31 niños.

Se han utilizado dos plataformas dinamométricas (Fig. 1) de $60 \times 45$ encajadas en el suelo de nuestro laboratorio de marcha. Sobre dichas plataformas caminaron los niños a su velocidad normal de marcha y un sistema informático procesó y analizó los datos referidos a los siguientes parámetros:

\section{Parámetros espaciales y temporales}

Longitud de paso

Tiempo de paso

Tiempo de doble apoyo

Tiempo de apoyo derecho e izquierdo

Coeficiente temporal entre el apoyo derecho e izquierdo

Indice temporal entre el doble apoyo y el apoyo derecho

Indice temporal entre el doble apoyo y el apoyo izqquierdo

Indice temporal entre el apoyo derecho y el apoyo total Indice temporal entre el apoyo izquierdo y el apoyo total

Indice temporal entre el doble apoyo y el apoyo total 


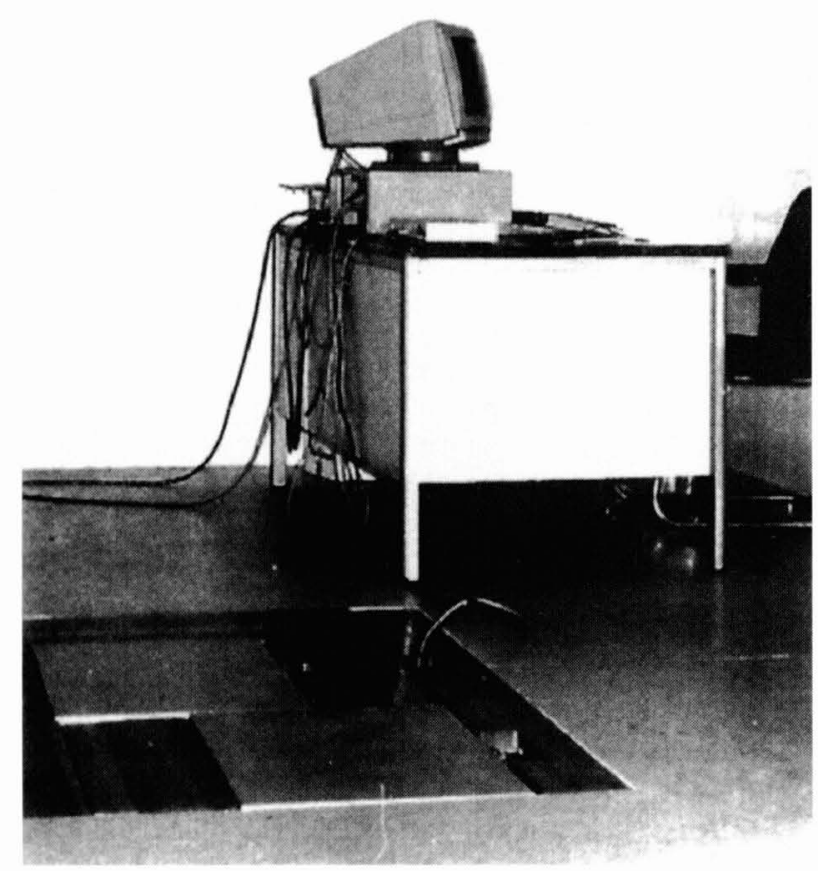

Fig. 1. Pasillo de marcha, plataforma de fuerza y equipo de registro.

\section{Parámetros cinéticos y su valor temporal}

Fuerza vertical de apoyo proximal derecho (FVAPD/P) Fuerza vertical de apoyo proximal izquierdo (FVAPI/P) Fuerza vertical de apoyo distal derecho (FVADD/P) Fuerza vertical de apoyo distal izquierdo (FVADI/P) Fuerza vertical media mínima derecha (FVMIND) Fuerza vertical media mínima izquierda (FVMINI) Fuerza máxima anterior derecha (FMAD) e izquierda (FMAI)

Fuerza máxima posterior derecha e izquierda (FMPDFMPI)

Fuerza máxima lateral derecha e izquierda (FMLDFMLI)

Fuerza máxima medial derecha e izquierda (FMMDFMMI)

\section{Indices de los tiempos de las fuerzas máximas}

Indice temporal fuerza vertical apoyo proximal derecha (ITFVAPD)

Indice temporal fuerza vertical apoyo proximal izquierda (ITFVAPI)

Indice temporal fuerza vertical apoyo distal derecha (ITFVADD)

Indice temporal fuerza vertical apoyo distal izquierda (ITFVADI)

\section{RESULTADOS}

La tabla I muestra los parámetros espaciales y temporales, en ella se aprecia que la longitud del paso es mayor en lo niños de 6 años, mientras que el tiempo de doble apoyo es mayor para el grupo de 5 años $(* * \mathrm{p}<0,01)$.

La tabla II muestra la magnitud de las fuerzas de reacción verticales. Podemos observar que la fuerva vertical de apoyo proximal derecha (FVAPD/P) y la fuerza vertical de apoyo distal izquierdo (FVADI/P) son mayores en los niños de 6 años $(* * p<0,01)$ y que la fuerza vertical de apoyo proximal izquierdo (FVAPI/P) y la fuerza vertical de apoyo distal derecho (FVADD/P) son también significativamente mayores en el grupo de niños de 6 años ( $* * * P<0,001)$. No habiendo diferencias estadísticamente significativas entre ambos grupos para el resto de los parámetros.

Nuestros resultados concuerdan con SCRUTON y cols. (1968); BECK y cols., (1981); NORLIN y cols., (1981); SUTHERLAND y cols., (1981) en cuanto a la longitud de paso. Asimismo NORLIN y cols., (1981) encuentra también que el tiempo de doble apoyo disminuye con la edad.

Nuestros resultados difieren de los de BECK y cols., (1981) en cuanto a las fuerzas de reacción del suelo. Este grupo de autores asegura que cada una de las tres componentes de las fuerzas de reacción normalizadas con el peso disminuyen al aumentar la edad (en el rango de 1 a 5 años) y luego se mantienen constantes.

\section{CONCLUSIONES}

1. Los parámetros de marcha obtenidos mediante plataformas de fuerza con distintos entre los niños de 5 y 6 años, lo que indica una evolución de la marcha en este periodo.

2. La longitud de paso es significativamente mayor en el grupo de 6 años.

3. La mayoría de las fuerzas de reacción del suelo en relación con el peso son significativamente mayores en el grupo de 6 años.

4. El tiempo de doble apoyo es mayor en el grupo de 5 años. 
TABLA 1

PARAMETROS TEMPORALES Y ESPACIALES

\begin{tabular}{llrrr}
\hline & & 5 AÑOS & 6 AÑOS \\
\hline \multirow{2}{*}{ L. PASO } & MEDIA & 425.839 & $* *$ & 6 AÑOS \\
& D.T. & 143.903 & & 103.593 \\
& E.S.M. & 23.846 & 18.606 \\
T. PASO & MEDIA & 596.129 & & 563.226 \\
& D.T. & 129.902 & 99.336 \\
& E.S.M. & 23.331 & & 17.841 \\
TDA & & & & \\
& MEDIA & 136.452 & $* *$ & 112.258 \\
& D.T. & 40.501 & 30.953 \\
& E.S.M. & 7.274 & 5.559 \\
TAD & & & \\
& MEDIA & 732.580 & 675.484 \\
& D.T. & 144.763 & 120.093 \\
& E.S.M. & 26.000 & 21.569 \\
TAI & & & \\
& MEDIA & 743.710 & 679.355 \\
& D.T. & 144.090 & 130.261 \\
& E.S.M. & 20.491 & 23.396 \\
& & & \\
TAD/TAI & MEDIA & 0.987 & 0.962 \\
& D.T. & 0.149 & 0.210 \\
& E.S.M. & 0.027 & 0.038 \\
\hline & & &
\end{tabular}

\section{BIBLIOGRAFIA}

Scrutton, D.S. and Robson, P. The gait of 50 Normal Children. Physiotherapy 1968; 54: 363-368.

Beck, R.J., Andriacchi, T.P., Kuo, K.N., Fermier, R.W. and Galante, J.O. Changes in the Gait Patterns of Growing children. The Journal of Bone and Joint Surgery 1981; 63 A.: 1452-1457.

Norlin, R., Ordenrick, P. and Sandlund, B. Development of Gait in The Normal children. J Pediat Orthop 1981; 1: 261266.
TABLA 2

MAGNITUD DE LAS FUERZAS VERTICALES

\begin{tabular}{|c|c|c|c|c|}
\hline & & AÑOS & & 6 AÑOS \\
\hline \multirow[t]{3}{*}{ FVAPD/P } & MEDIA & 1.001 & $\star *$ & 1.146 \\
\hline & D.T. & 0.203 & & 0.224 \\
\hline & E.S.M. & 0.036 & & 0.040 \\
\hline \multirow[t]{3}{*}{ FVAPI/P } & MEDIA & 1.075 & 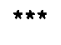 & 1.234 \\
\hline & D.T. & 0.127 & & 0.204 \\
\hline & E.S.M. & 0.023 & & 0.037 \\
\hline \multirow[t]{3}{*}{ FVADD/P } & MEDIA & 1.050 & $\star \star \star \star$ & 1.143 \\
\hline & D.T. & 0.068 & & 0.122 \\
\hline & E.S.M. & 0.012 & & 0.022 \\
\hline \multirow[t]{3}{*}{ FVADI/P } & MEDIA & 1.017 & ** & 1.117 \\
\hline & D.T. & 0.094 & & 0.144 \\
\hline & E.S.M. & 0.017 & & 0.026 \\
\hline \multirow[t]{3}{*}{ FVMIND/P } & MEDIA & 0.832 & & 0.814 \\
\hline & D.T. & 0.103 & & 0.169 \\
\hline & E.S.M. & 0.018 & & 0.029 \\
\hline \multirow[t]{3}{*}{ FVMINI/P } & MEDIA & 0.788 & & 0.815 \\
\hline & D.T. & 0.081 & & 0.193 \\
\hline & E.S.M. & 0.015 & & 0.034 \\
\hline
\end{tabular}

Sutherland, D.H., Olsmer, R., Cooper, L. and Woo, S.L.Y. The Development of Mature Gait. The Journal of Bone and Joint Surgery 1980; 62-A(3): 336-353.

Hetzog, W., Nigg, B.M., Read, L.S., Olssen, E. Asymmetries in ground reaction force patterns in normal human gait. Medicine and Science in Sports and Exercise 1989; 21(1): 110-114.

Begg, R.K., Wytch, R., Major, R.E. A microcomputer-based video vector system from clinical gait analysis. J Biomed Eng 1990; 12: 383-388. 